\title{
Activation of Nrf2 Signaling by Apelin Attenuates Renal Ischemia Reperfusion Injury in Diabetic Rats
}

This article was published in the following Dove Press journal:

Diabetes, Metabolic Syndrome and Obesity: Targets and Therapy

\author{
Xiaobo Zhang \\ Ying Zhu \\ Ying Zhou \\ Bingru Fei
}

Nephrology Department, The Affiliated Huaian No. I People's Hospital of Nanjing Medical University, Huaian, Jiangsu Province 223300, People's Republic of China
Correspondence: Xiaobo Zhang The Affiliated Huaian No. I People's Hospital of Nanjing Medical University, Number I Huanghe Xi Lu, Huaiyin

District, Huaian, Jiangsu Province 223300,

People's Republic of China

Email zhangxiaobo2020@yeah.net
Objective: Renal ischemia/reperfusion (I/R) injury is commonly seen in diabetic patients. Apelin has been demonstrated to protect against renal I/R injury, whereas detailed modulatory mechanisms by which Apelin exerts its role in renal $I / R$ injury in diabetic patients remain unclarified. This research aimed to probe the functional molecules under the regulation of Apelin in renal I/R injury in diabetic rats.

Materials and Methods: First, animal models were established for subsequent assays. Biochemical kits measured the serum levels of blood urea nitrogen (BUN) and serum creatinine (SCR), and hematoxylin and eosin (H\&E) staining examined the histopathological changes of kidney tissues. Inflammatory factors containing tumor necrosis factor alpha (TNF- $\alpha$ ), interleukin $1 \beta$ (IL-1 $\beta$ ), interleukin 6 (IL-6) and monocyte chemoattractant protein1 (MCP-1) were tested through enzyme-linked immunosorbent assay (ELISA) and reverse transcription-quantitative polymerase chain reaction (RT-qPCR), respectively. Reactive oxygen species (ROS) levels in the serum and kidney tissues were separately assessed by specific ROS kits. Cell apoptosis was further estimated through terminal deoxynucleotidyl transferasemediated dUTP nick end labeling (TUNEL) and Western blot analysis. Eventually, the influences of Apelin on nuclear factor erythroid 2-related factor (Nrf2) and its downstream genes were explored via Western blot analysis and immunohistochemistry (IHC).

Results: In the present study, Apelin ameliorated the damage to renal function and histological structure, decreased levels of inflammatory factors and ROS, and hampered cell apoptosis in renal I/R injury of diabetic rats. Moreover, Apelin could elevate the levels of Nrf2 and downstream genes which were decreased under renal I/R injury.

Conclusion: These data indicated that Apelin inhibited renal $I / R$ injury through regulating Nrf2 signaling in diabetic rats, which might shed new light on the treatment of renal I/R injury in diabetic patients.

Keywords: renal ischemia/reperfusion injury, diabetes, Apelin, Nrf2 signaling

\section{Introduction}

Diabetes is a metabolic disease featured by hyperglycemia. People with diabetes have high blood glucose levels owing to defects in insulin secretion and/or insulin action. ${ }^{1,2}$ Type 1 diabetes and type 2 diabetes are the main types. Type 1 diabetes is characterized by a complete or nearly complete insulin deficiency and type 2 diabetes, the most common type of diabetes, is characterized by insulin resistance and a dysfunction of insulin synthesis and release by pancreatic $\beta$-cells. ${ }^{3,4}$ Increasing reports have illustrated that diabetes patients have a higher risk of cardiovascular diseases (CVDs), ${ }^{5,6}$ which can, in turn, promote the progression and generate adverse outcomes. ${ }^{7}$ Due to the original damage to renal blood flow, after I/R injury, hypoxia-sensitive renal tissue and 
cells produce excessive ROS in an explosive manner, resulting in cell DNA damage and apoptosis and necrosis of cells in renal tissue. In addition, free radicals are produced, which may lead to the release of inflammation mediators and cell apoptosis. As we know, kidneys are hyperperfusion organs and sensitive to ischemia/reperfusion $(\mathrm{I} / \mathrm{R})$. I/R injury is a complex process that can occur in shock patients, kidney trauma, and kidney transplantation. ${ }^{8,9}$ As the incidence of type 2 diabetes continues to increase, the incidence of $I / R$ injury in diabetic patients increases significantly, and the renal function is obviously impaired, which can lead to acute renal failure in severe cases. ${ }^{10}$ Thus, renal injury is often caused by $\mathrm{I} / \mathrm{R}$ treatment in diabetes. ${ }^{11,12}$ However, studies on the renal I/R injury in diabetes are still inadequate nowadays.

Apelin gene (APLN) encodes a 77-amino acid preproapelin, which is later cleaved into 13-, 17-, and 36-amino acid peptides from the C-terminus. Apelin is a peptide that acts as a ligand for angiotensin I receptor-related protein $\mathrm{J}$ receptor (APJ), a member of GPCR (G-proteincoupled receptor) family. ${ }^{13}$ APJ is the only endogenous receptor for apelin that mediates signal transduction via $\mathrm{G}$ protein. Mounting evidence has reported the protective role of Apelin in renal injury, ${ }^{14}$ ischemic stroke, ${ }^{15}$ lung injury, ${ }^{16}$ and so on. The precise mechanism underlying Apelin in renal $\mathrm{I} / \mathrm{R}$ injury in diabetes needs further investigation.

Nuclear factor erythroid 2-related factor 2 (Nrf2), a transcription factor, is essential for downstream gene regulation in mammals. Nrf2 can exert suppressive functions in plenty of diseases. For example, activation of Nrf2 in osteoblasts represses osteoclastogenesis through repression of IL-6. ${ }^{17}$ MiR-200a ameliorates doxorubicinproduced cardiotoxicity by increasing Nrf2 levels in mice. ${ }^{18}$ Besides, astaxanthin improves the lipopolysaccharidescaused subfertility of the mouse through Nrf2/HO-1 antioxidant pathway. ${ }^{19}$ It is also reported that melatonin attenuates acute kidney $\mathrm{I} / \mathrm{R}$ injury in diabetic rats by activating SIRT1/Nrf2/HO-1 pathway. $^{20}$ Nevertheless, the association between Apelin and Nrf2 in renal I/R injury in diabetic rats has never been explored yet.

In the current research, we established the animal models to induce renal injury. We observed the changes of kidney function, histological structure, inflammatory cytokines, ROS levels and cell apoptosis. The effects of Apelin on renal $\mathrm{I} / \mathrm{R}$ injury in diabetic rats were also examined.

\section{Materials and Methods}

\section{Animal Models and Grouping}

In this study, male Sprague-Dawley rats $(\mathrm{N}=25,180-200 \mathrm{~g})$ from Hunan SJA Laboratory Animal Co., Ltd (Changsha, China) were used. These experimented rats were maintained in the condition of $12 \mathrm{~h}$ light/dark cycle, $25^{\circ} \mathrm{C}$ and $30-45 \%$ humidity. Following the principles in the NIH Guide for the Care and Use of Laboratory Animals, all experimental procedures were accomplished, which was also permitted by the Laboratory Animal Ethics Committee at The Affiliated Huaian No.1 People's Hospital of Nanjing Medical University.

Rats were given 1 week for acclimation and then allocated to five different groups: Control, $\mathrm{Db}, \mathrm{Db}+\mathrm{I} / \mathrm{R}, \mathrm{Db}+\mathrm{I} / \mathrm{R}+\mathrm{NC}$, and $\mathrm{Db}+\mathrm{I} / \mathrm{R}+\mathrm{AP}$, where $\mathrm{Db}$ represents diabetes, $\mathrm{I} / \mathrm{R}$ represents ischemia/reperfusion, and AP represents Apelin. Firstly, rats in the control group were fed standard rat chow and rats in the $\mathrm{Db}$ group were fed a high-fat diet (HFD) as previously described. ${ }^{21}$ In the sixth week, control rats and $\mathrm{Db}$ rats were, respectively, injected with saline and streptozotocin (STZ) $(30 \mathrm{mg} / \mathrm{kg})$. Three days post STZ injection, a glucometer was employed to measure fasting blood glucose (FBG) level. Rats with FBG levels of more than $11.1 \mathrm{mmol} / \mathrm{L}$ were confirmed as type 2 diabetes rats. Secondly, diabetic rats were provided with normal water and diet ad libitum for another 2 months before renal I/R. Rats were anesthetized with 3\% pentobarbital sodium in a dose of $50 \mathrm{mg} / \mathrm{kg}$ before the midline laparotomy. Thereafter, the right kidney was resected and the left renal pedicle was clamped in a non-traumatic way for about 45 mins. When kidney color ranged from red to pale, ischemia was successfully induced. After clamp was removed, kidney color changed back to red, suggesting that blood flow restoration was successful. After that, rats were put on a heating pad at $37^{\circ} \mathrm{C}$ until their consciousnesses were recovered. Post $24 \mathrm{~h}$ of reperfusion, rats were allowed for recovery for $3 \mathrm{~d}$ before sacrifice. Tail vein injection of lentivirus vectors or Apelin lentivirus was performed in rats. After the sacrifice of rats, the blood samples were gathered for renal function examination, and left kidneys were gained and sliced into two pieces for future use.

\section{Biochemical Analysis and Histological Examination}

Blood samples were collected through the abdominal aorta. Two indexes, blood urea nitrogen (BUN) and serum creatinine (SCR), were estimated with kits procured from Nanjing Jiancheng Bioengineering Institute (Nanjing, China). 
Isolated kidneys were fastened in $10 \%$ formalin for $1 \mathrm{~d}$ and subsequently embedded in paraffin. Sections $(5 \mu \mathrm{m})$ of kidney tissues were cut from paraffinembedded samples and dyed with hematoxylin and eosin (H\&E). In subsequence, slides were evaluated via using a light microscope (Nikon E600W, Japan). Expert investigators with no idea of the experiment and data evaluated each slide for degrees of histological damage (none, mild, moderate and severe).

\section{Enzyme-Linked Immunosorbent Assay (ELISA)}

Separation of serum by 10 min of centrifugation at 4000 rpm was performed, followed by collection and reposition at $-80^{\circ} \mathrm{C}$. Serum levels of pro-inflammatory cytokines comprising of tumor necrosis factor (TNF)- $\alpha$, interleukin (IL)-1 $\beta$, IL-6 and monocyte chemoattractant protein-1 (MCP-1) were analyzed through the application of ratspecific ELISA kits strictly following the protocols of the manufacturer (Elabscience Biotechnology Co., Ltd, Wuhan, China). The optical density (OD) values were assessed via an automatic microplate reader (Thermo Fisher Scientific, Waltham, MA, USA). Pro-inflammatory cytokines in kidney tissues were also investigated through ELISA. Before the assay, the tissue homogenate was centrifugated $(5000 \times \mathrm{g})$ for 5 to $10 \mathrm{~min}$ and stored at $-80^{\circ} \mathrm{C}$.

\section{Reverse Transcription-Quantitative PCR (RT-qPCR)}

The mRNA expression of TNF- $\alpha$, IL-1 $\beta$, IL- 6 and MCP-1 in kidney tissues was explored utilizing RT-qPCR. Trizol Reagent (Takara, Dalian, China) was utilized to harvest total RNA, followed by cDNA synthesis by Frist Strand cDNA Synthesis Kit (Takara, Dalian, China), as guided by the instructions. Thereafter, relative mRNA expression was quantified with the help of SYBR Green qPCR Master Mix kit (Thermo Scientific, Wilmington, DE) in triplicates. The parameters of RT-qPCR amplification reaction were as follows: 40 cycles of $95^{\circ} \mathrm{C}$ for just $10 \mathrm{~s}$ (denaturation) and $72^{\circ} \mathrm{C}$ for about $30 \mathrm{~s}$ (annealing/extension). Other mRNA expression levels were normalized to that of GAPDH.

\section{Detection of Reactive Oxygen Species (ROS)}

ROS levels in the serum and kidney tissues were, respectively, assessed. In detail, serum ROS levels were measured utilizing Fenton's reaction and Griess reagent chromogenic method, and those in kidney tissues were determined with the DCFH-DA method as directed by the manufactures. All kits were procured from the Nanjing Jiancheng Bioengineering Institute (Nanjing, China).

\section{TUNEL Staining}

Cell apoptosis was estimated via TUNEL assay. Before the assay, paraffin-embedded sections suffered deparaffinage and rehydration. In accordance with the guidebook, TUNEL assay with an InSitu Cell Death Detection Kit (Roche, Mannheim, Germany) was carried out to detect apoptotic cells. TUNEL-positive cells at six random areas were calculated through an Olympus BX60 microscope that was equipped with a digital CCD. Apoptosis rate was figured out as follows: number of TUNEL-positive nuclei/total number of cells per field $\times 100 \%$.

\section{Western Blot Analysis}

Kidney tissues were subjected to sonication in pre-cold RIPA buffer (Beyotime, Haimen, China) for the late quantification of protein concentrations by the BCA method. 20-80 $\mu \mathrm{g}$ of protein was isolated with the employment of SDS-PAGE, which was sequentially transferred to PVDF membranes for further immunodetection. Next, the membranes were blocked with defatted milk at indoor temperature. Culture with primary antibodies for one night at $37^{\circ} \mathrm{C}$ and secondary goat-anti-mouse horseradish peroxidase (HRP)-conjugated antibody for 2 $\mathrm{h}$ were sequentially performed. Eventually, the intensity of protein bands was tested by enhanced chemiluminescence (ECL) reagents (Thermo Fisher Scientific) and evaluated via the use of Quantity One 1-D Analysis Software. Primary antibodies, containing Bax, cleaved caspase-3, Bcl2, Nrf2, catalase (CAT), NAD(P)H dehydrogenase, quinone 1 (NQO1), superoxide dismutase 1 (SOD1), glutathione synthetase (GSS), glutamatecysteine ligase modifier subunit (GCLM), glutamatecysteine ligase catalytic (GCLC) and GAPDH, were purchased from Abcam.

\section{Immunohistochemistry (IHC)}

Paraffin-embedded samples were cut into different sections, deparaffinized, and rehydrated. Next, they were blocked using methanolic 3\% hydrogen peroxide. Citrate buffer was applied for antigen retrieval. Post the incubation with the primary antibody against anti-Nrf2 and the 
HRP-labelled goat-anti-mouse antibody, the detection was implemented with the Iview DAB detection kit (Catalog number 760-091, Ventana, Tucson, AZ).

\section{Statistical Analysis}

Each assay was conducted at least three times. Experimental data analyzed via GraphPad Prism Software (GraphPad software, Inc., La Jolla, CA) were expressed as the mean \pm standard deviation (SD). The differences among groups were dissected utilizing one-way ANOVA. P value below 0.05 meant that statistics were significant.

\section{Results}

\section{Apelin Reduced Renal I/R Injury in Diabetic Rats}

Renal I/R injury of diabetic rats were induced for this study. We firstly detected the serum levels of BUN and SCR, two indexes of kidney function. The results revealed that in the serum of Db rats, BUN and SCR levels were significantly increased. In comparison with $\mathrm{Db}$ group, I/R treatment further elevated the serum levels of BUN and SCR, which was decreased when Apelin was added (Figure 1A). H\&E staining found that histological injury induced in $\mathrm{Db}$ group was further promoted after I/R treatment, and this promotion was inhibited

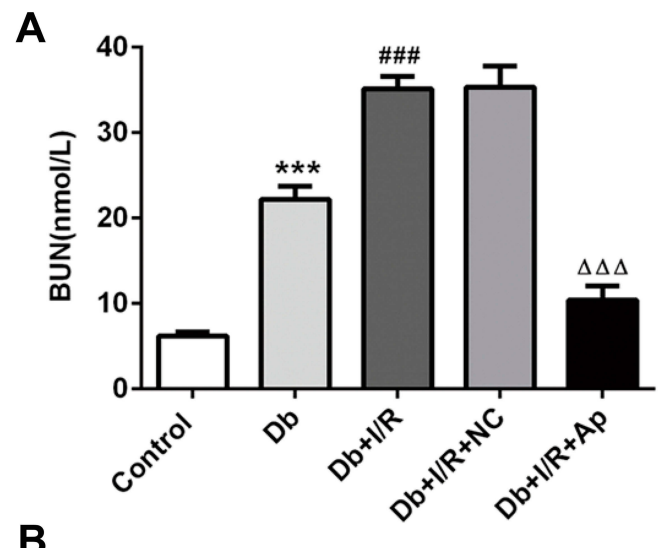

B
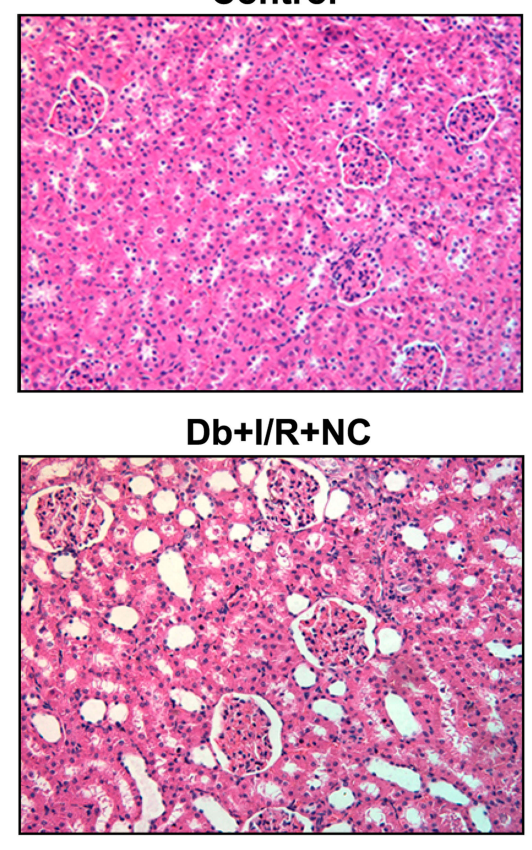
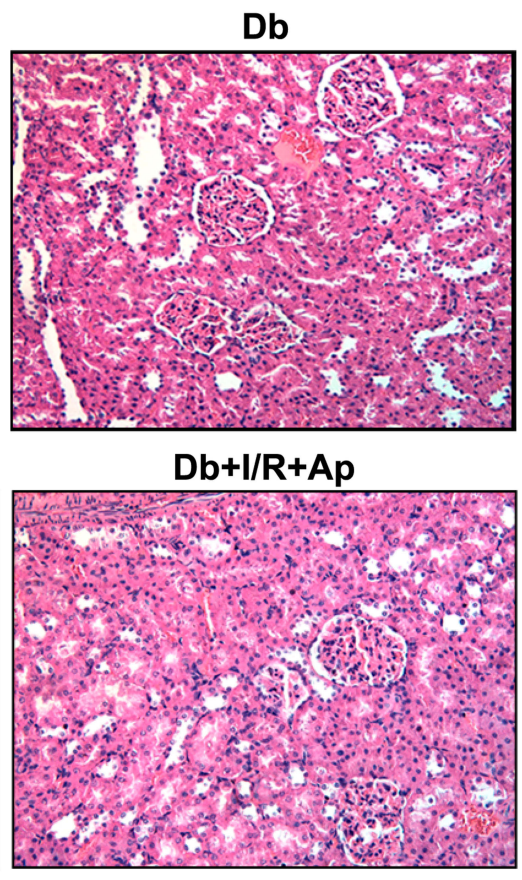

Db
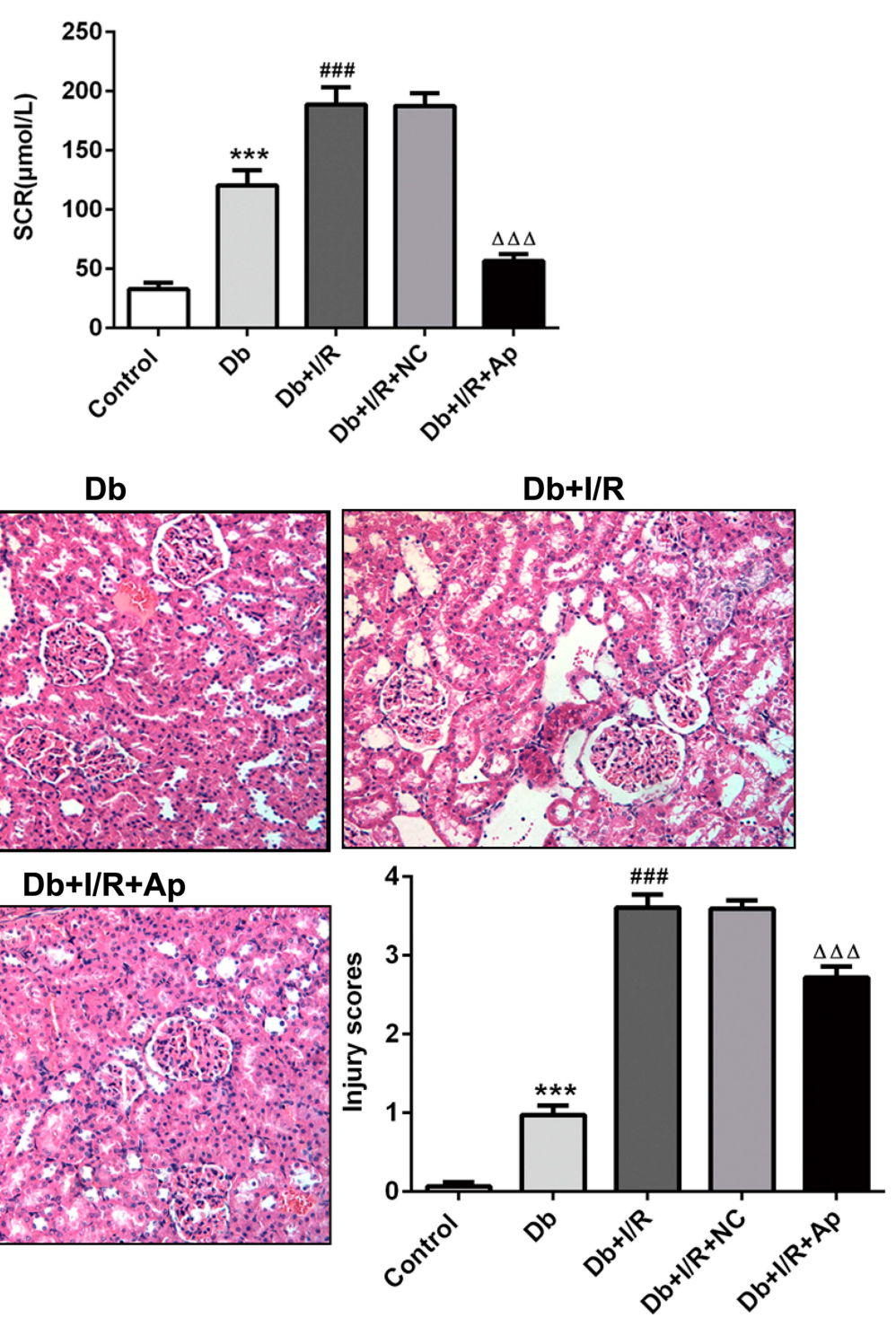

Figure I Effects of Apelin on the function and histological structure of kidneys. (A) Relative biochemical kits for detection of BUN and SCR levels in the serum of rats in five groups. (B) H\&E staining of histopathological changes in five groups of kidney tissues. ${ }^{* * *} \mathrm{P}<0.00 \mathrm{I}$ vs Control; ${ }^{\#} \mathrm{P}<0.00 \mathrm{I}$ vs $\mathrm{Db} ;{ }^{\Delta \Delta \Delta} \mathrm{P}<0.00 \mathrm{I}$ vs $\mathrm{Db}+\mathrm{I} / \mathrm{R}+\mathrm{NC}$. 
when Apelin was up-regulated (Figure 1B). These data demonstrated that Apelin could inhibit renal $\mathrm{I} / \mathrm{R}$ injury in diabetic rats.

\section{Apelin Decreased the Levels of Inflammatory Factors in the Serum and Kidney Tissues}

We further examined the inflammation responses in different groups. ELISA and RT-qPCR were, respectively, applied to test the levels of inflammatory cytokines, such as TNF-a, IL-1 $\beta$, IL-6 and MCP-1. It was observed in ELISA assay that TNF-a, IL-1 $\beta$, IL- 6 and MCP-1 were dramatically raised in $\mathrm{Db}$ group, and $\mathrm{I} / \mathrm{R}$ treatment accelerated the elevation. After Apelin addition, TNF-a, IL-1 $\beta$, IL-6 and MCP-1 were distinctly lessened (Figure 2A). Moreover, RT-qPCR experiment discovered that $\mathrm{Db}$ increased levels of inflammatory factors were improved when $I / R$ treatment was performed. And the promotion was repressed in response to Apelin overexpression (Figure 2B). Taken together, boosted inflammation response in renal $\mathrm{I} / \mathrm{R}$ injury of rats with diabetes was inhibited when rats were injected with Ad-Apelin.

\section{Apelin Lowered ROS Levels in the Serum and Kidney Tissues}

Then, ROS levels in five different groups were separately measured. Serum levels of ROS from Db rats were obviously high compared with control rats. I/R treatment enhanced the ROS levels activated by induction of diabetes; however, this phenomenon was markedly restrained with the addition of Apelin. Similarly, in kidney tissues, elevated ROS levels in $\mathrm{Db}$ group were facilitated after I/R injury, and compared with $\mathrm{Db}+\mathrm{I} / \mathrm{R}+\mathrm{NC}$ group, ROS levels were significantly suppressed in $\mathrm{Db}+\mathrm{I} / \mathrm{R}+\mathrm{Ap}$ group (Figure $2 \mathrm{C}$ ). These findings told us that promoted ROS levels in diabetes I/R-caused renal injury was lowered after Apelin treatment.

\section{Apelin Decreased the Stimulated Cell Apoptosis in Kidney Tissues}

Cell apoptosis was subsequently examined. In TUNEL assay, we observed that cell apoptosis of Db group was dramatically activated compared to control group, and I/R injury further accelerated the apoptosis. Apelin treatment hampered the induced cell apoptosis (Figure 3A and B). Results of Western blot affirmed these findings. Protein levels of Bax
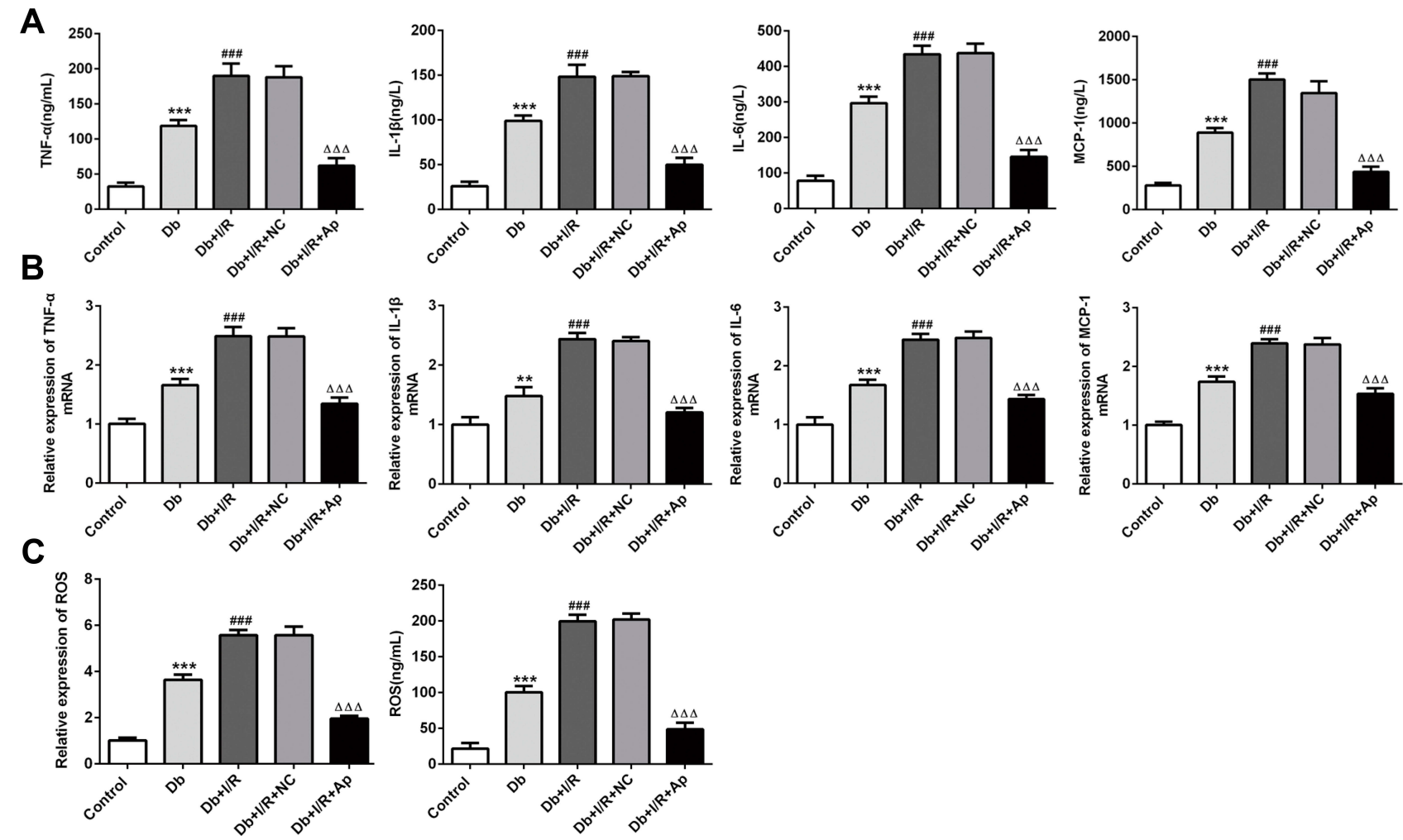

Figure 2 Effects of Apelin on inflammatory cytokines and oxidative stress. (A, B) TNF-a, IL-I $\beta$, IL-6 and MCP-I levels were separately estimated via the usage of ELISA and

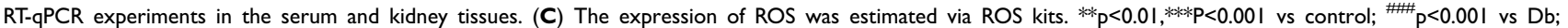
$\Delta \Delta \Delta \mathrm{P}<0.00 \mathrm{I}$ vs $\mathrm{Db}+\mathrm{I} / \mathrm{R}+\mathrm{NC}$. 

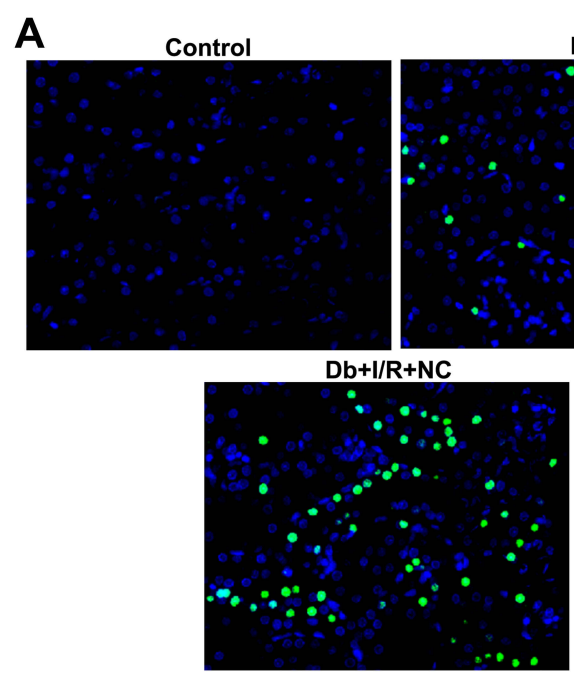

C
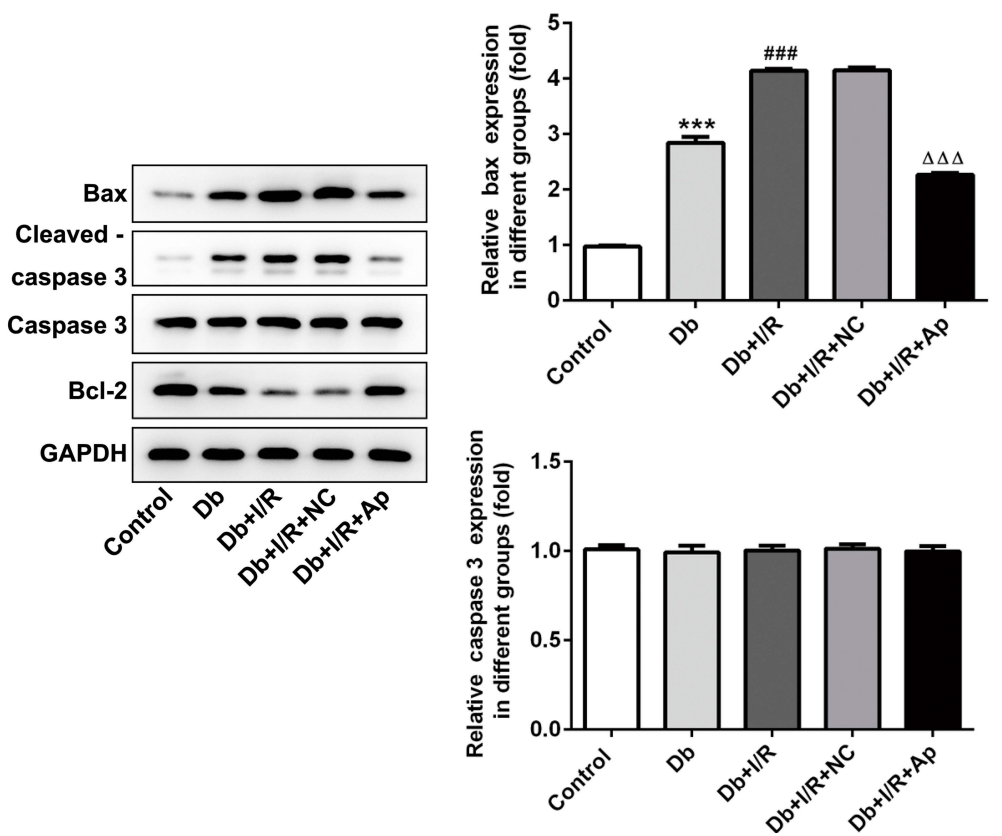

B

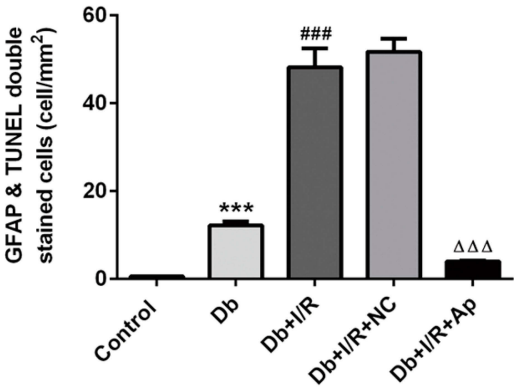

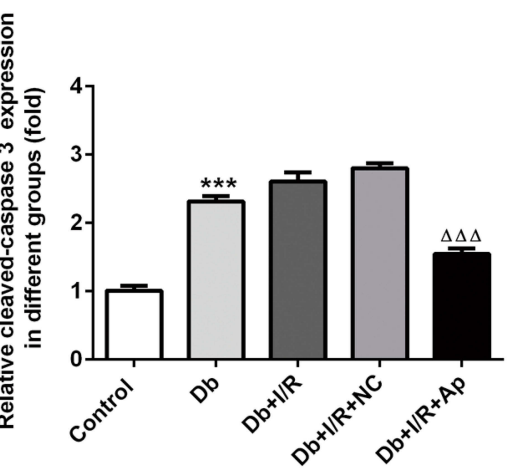

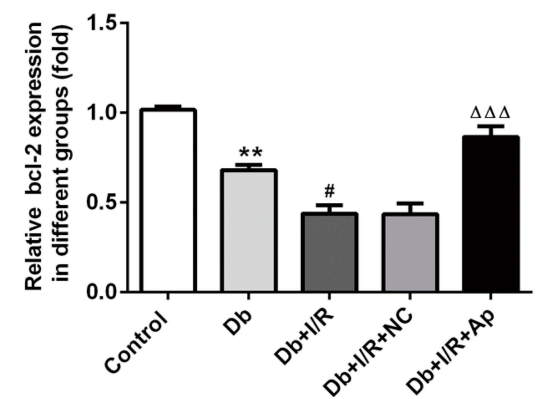

Figure 3 Impacts of Apelin on the apoptosis. (A) TUNEL assay was utilized to measure cell apoptosis in five groups. (B) Statistical analysis of apoptosis. (C) Western blot assessed the protein levels of apoptosis-related proteins. ${ }^{* *} \mathrm{p}<0.0 \mathrm{I},{ }^{* * *} \mathrm{p}<0.00 \mathrm{I}$ vs control; ${ }^{*} \mathrm{p}<0.05,{ }^{\#} \mathrm{p}<0.00 \mathrm{I}$ vs $\mathrm{Db} ;{ }^{\Delta \Delta \Delta} \mathrm{p}<0.00 \mathrm{I}$ vs $\mathrm{Db}+\mathrm{l} / \mathrm{R}+\mathrm{NC}$.

and cleaved caspase- 3 were increased but $\mathrm{Bcl} 2$ protein levels were decreased after diabetes induction, which was strengthened with I/R treatment. Thereafter, Apelin lowered the elevated protein levels of Bax and cleaved caspase- 3 and enhanced the lessened protein levels of $\mathrm{Bcl} 2$ (Figure 3C). In summary, induced cell apoptosis in renal I/R injury was hindered in the context of Apelin.

\section{Apelin Activated Nrf2 Signaling During Renal I/R Injury in Diabetic Rats}

To investigate the regulatory mechanism under Apelin in renal $\mathrm{I} / \mathrm{R}$ injury, we used Western blot to detect the expression changes of Nrf2 and its downstream factors. Protein levels of
Nrf2, CAT, NQO1, SOD1, GSS, GCLM and GCLC were markedly reduced under diabetes and $\mathrm{I} / \mathrm{R}$ treatment, alone and in combination, whereas the supplement of Apelin reversed these decreases (Figure 4). IHC assay displayed that $\mathrm{Nrf} 2$ expression was lessened in Db group, and the reduction was enhanced after I/R treatment. However, Apelin treatment rescued the down-regulation of Nrf2 (Figure 5). All in all, Apelin activated Nrf2 signaling to ameliorate diabetes I/ R-activated renal injury.

\section{Discussion}

Recently, increasing reports have discussed about the renal ischemia/reperfusion (I/R) injury in diabetes. For instance, 


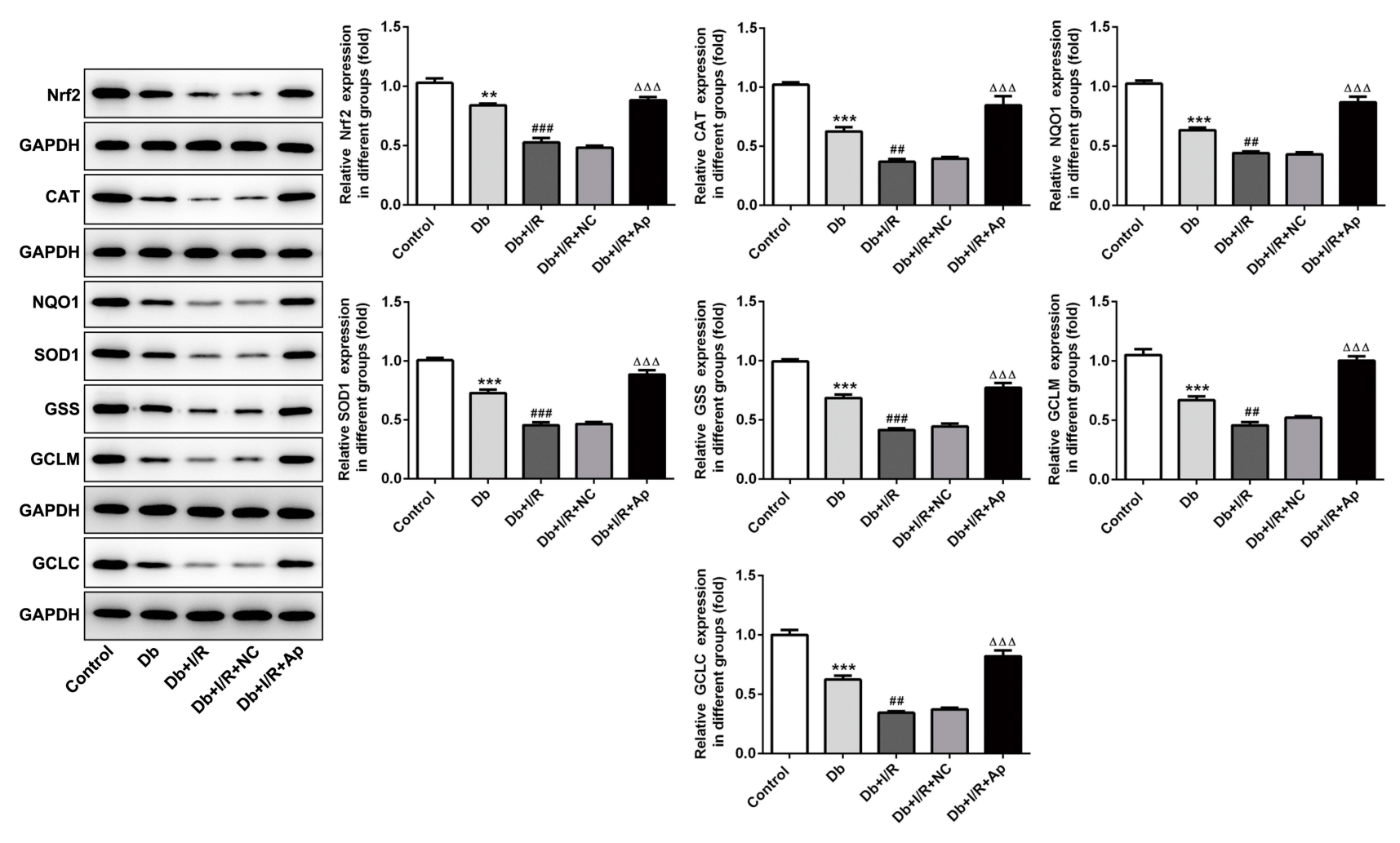

Figure 4 Apelin had a promotive effect on Nrf2 and its downstream genes. Western blot detection of Nrf2, CAT, NQOI, SODI, GSS, GCLM and GCLC levels under five treatments. ${ }^{* *} \mathrm{p}<0.01,{ }^{*} * * *_{\mathrm{p}}<0.001$ vs control; ${ }^{\#} \mathrm{p}<0.01,{ }^{\ldots+\ldots} \mathrm{p}<0.001$ vs $\mathrm{Db} ;{ }^{\Delta \Delta \Delta} \mathrm{p}<0.001$ vs $\mathrm{Db}+\mathrm{l} / \mathrm{R}+\mathrm{NC}$.
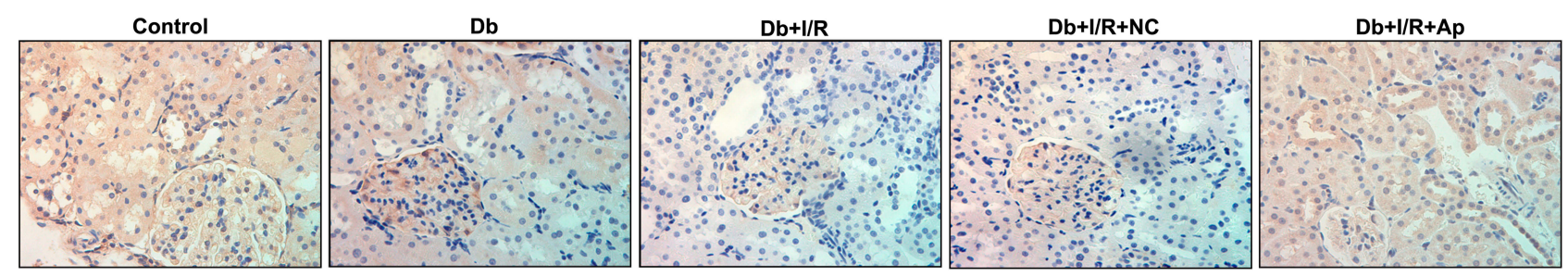

Figure 5 Apelin had a promotive effect on Nrf2. IHC analysis of Nrf2 expression changes under five kinds of treatments.

calcium-sensing receptor plays a stimulative role in streptozotocin-caused diabetic rats with renal $\mathrm{I} / \mathrm{R}$ injury. ${ }^{22} \mathrm{P} 2 \mathrm{X} 7$ receptor depletion prevents against ischemic acute kidney injury of mice. ${ }^{23}$ Captopril has protective effects on diabetic rats along with $\mathrm{I} / \mathrm{R}$ renal injury. ${ }^{24}$ Both telmisartan and pioglitazone develop renoprotective functions in I/R-induced kidney damage in diabetic rats. ${ }^{25}$ In view of the complexity of $\mathrm{I} / \mathrm{R}$-induced renal damage in diabetic rats, present researches are still inadequate and we need to conduct more researches to further comprehend the pathogenesis of renal $\mathrm{I} / \mathrm{R}$ injury in diabetes.

Commonly, renal function damage, histopathological changes, ROS damage, inflammation response, and cell apoptosis are pivotal phenotypes of renal damage, which has been yet reported by several researches. ${ }^{26-28}$ Dabrafenib can exert a protective role in renal $\mathrm{I} / \mathrm{R}$ injury via improving renal function, as well as reducing histological injury, inflammation and cell death. ${ }^{29}$ MAPK-ERK-CREB pathway mediates renal tubular inflammation response and cell death under hypoxiareoxygenation injury. ${ }^{30}$ Also, targeting ROS and cPLA2/ COX2 can decrease renal damage to obese mice with endotoxemia. ${ }^{31}$ Hence, further research paid much attention to the changes of cellular functions under various treatments.

In this work, we aimed to investigate the Apelin role and the downstream molecules under Apelin in renal $\mathrm{I} / \mathrm{R}$ injury of diabetic rats. Apelin has been illustrated to repress renal $I / R$ injury by affecting multiple cellular activities. For example, Apelin hormone prevents renal I/R-activated oxidative damage in rats. ${ }^{13}$ Apelin protects against acute renal injury through 
repression of TGF- $\beta 1 .^{32}$ Moreover, Apelin and leptin have protective impacts on renal functions following renal I/R damage. ${ }^{33}$ However, up to now, there is no study about the function of Apelin in renal $\mathrm{I} / \mathrm{R}$ damage in diabetes. In the present paper, we divided rats into five groups to observe the effect of Apelin on renal I/R injury in diabetic rats. The results indicated that renal function damage, histological injury, and the improved levels of inflammation response, ROS and apoptosis occurred under renal $\mathrm{I} / \mathrm{R}$ injury in diabetic rats were all reversed with the addition of Apelin.

Further, we sought to probe the exact mechanism of Apelin in diabetic renal I/R injury. Nuclear factor erythroid 2-related factor $2(\mathrm{Nrf} 2)$ is a researched protector against diabetes I/R-caused renal injury. Nrf2 is found to be upregulated under naringin and trimetazidine that develop protective properties in the remote effect of acute renal injury by inhibition of oxidative stress and myocardial injury. ${ }^{34}$ Moreover, inhibition of glycogen synthase Kinase-3 $\beta$ activates Nrf2/HO-1 axis to attenuate renal I/R damage in rats suffering from diabetes. ${ }^{35}$ Thus, we supposed that Apelin might exert its inhibitory effect on renal I/R damage in diabetic rats via stimulation of Nrf2. Our current study revealed that protein levels of $\mathrm{Nrf} 2$ and its downstream antioxidative enzymes were reduced in response to renal $\mathrm{I} / \mathrm{R}$ injury of diabetic rats, which were recovered with the up-regulation of Apelin. IHC results confirmed the expression changes of Nrf2 among different groups. It was the first time that the association between Apelin and Nrf2 in I/R-induced renal damage in diabetic rats was uncovered.

\section{Conclusion}

In conclusion, this study disclosed that Apelin might protect renal from $\mathrm{I} / \mathrm{R}$ injury in diabetic rats by modulating Nrf2 and its downstream genes. These data would offer a promising treatment method for diabetes patients with renal $\mathrm{I} / \mathrm{R}$ injury.

\section{Data Sharing Statement}

The analyzed data sets generated during the present study are available from the corresponding author on reasonable request.

\section{Disclosure}

The authors declare that they have no competing interests.

\section{References}

1. Li M, Ikehara S. Bone marrow stem cell as a potential treatment for diabetes. J Diabetes Res. 2013;2013:329596. doi:10.1155/2013/329596

2. Costa PZ, Soares R. Neovascularization in diabetes and its complications. Unraveling the angiogenic paradox. Life Sci. 2013;92 (22):1037-1045. doi:10.1016/j.lfs.2013.04.001

3. Rocca B, Rubboli A, Zaccardi F. Antithrombotic therapy and revascularisation strategies in people with diabetes and coronary artery disease. Eur J Prev Cardiol. 2019;26(2_suppl):92-105. doi:10.1177/ 2047487319880045

4. Gao H, Yang Y, Deng J, Liang J, Zhang W, Feng X. A systematic review and meta-analysis on the efficacy and safety of traditional Chinese patent medicine Jinqi Jiangtang Tablet in the treatment of type 2 diabetes. Complement Ther Med. 2019;47:102021. doi:10.1016/j.ctim.2019.01.016

5. Palumbo PJ, Elveback LR, Chu C-P, Connolly DC, Kurland LT. Diabetes mellitus: incidence, prevalence, survivorship, and causes of death in Rochester, Minnesota, 1945-1970. Diabetes. 1976;25 (7):566-573. doi:10.2337/diab.25.7.566

6. Harris-Hayes M, Schootman M, Schootman JC, Hastings MK. The role of physical therapists in fighting the type 2 diabetic epidemic. J Orthop Sports Phys Ther. 2019;1-35.

7. Han D, Rozanski A, Gransar H, et al. Myocardial ischemic burden and differences in prognosis among patients with and without diabetes: results from the multicenter international REFINE SPECT registry. Diabetes Care. 2019;43(2):453-9.

8. Yao X, Tan G, He C, et al. Hydrogen sulfide protects cardiomyocytes from myocardial ischemia-reperfusion injury by enhancing phosphorylation of apoptosis repressor with caspase recruitment domain. Tohoku J Exp Med. 2012;226(4):275-285. doi:10.1620/tjem.226.275

9. Moeini M, Nematbakhsh M, Fazilati M, et al. Protective role of recombinant human erythropoietin in kidney and lung injury following renal bilateral ischemia-reperfusion in rat model. Int J Prev Med. 2013;4(6):648-655.

10. Kantharidis P, Wang B, Carew RM, Lan HY. Diabetes complications: the microRNA perspective. Diabetes. 2011;60(7):1832-1837. doi:10. 2337/db11-0082

11. Okada H, Tanaka M, Yasuda T, et al. Decreased microcirculatory function measured by perfusion index is a novel indicator of diabetic kidney disease in patients with type 2 diabetes. J Diabetes Investig. 2019.

12. Tang Y, Tang J, Qian P, et al. Recombinant human erythropoietin restrains oxidative stress in streptozotocin-induced diabetic rats exposed to renal ischemia reperfusion injury. Transplant Proc. 2019;51(6):2076-2080. doi:10.1016/j.transproceed.2019.03.023

13. Bircan B, Çakır M, Kırbağ S, Gül HF. Effect of apelin hormone on renal ischemia/reperfusion induced oxidative damage in rats. Ren Fail. 2016;38(7):1122-8.

14. Kim JS, Yang JW, Han BG, Kwon HJ, Kim JH, Choi SO. Protective role of apelin against cyclosporine-induced renal tubular injury in rats. Transplant Proc. 2017;49(6):1499-1509. doi:10.1016/j.transproceed. 2017.03.080

15. Qiu J, Wang X, Wu F, et al. Low dose of apelin-36 attenuates ER stress-associated apoptosis in rats with ischemic stroke. Front Neurol. 2017;8:556. doi:10.3389/fneur.2017.00556

16. Zhang L, Li F, Su X, et al. Melatonin prevents lung injury by regulating apelin 13 to improve mitochondrial dysfunction. Exp Mol Med. 2019;51(7):73. doi:10.1038/s12276-019-0273-8

17. Narimiya T, Kanzaki H, Yamaguchi Y, et al. Nrf2 activation in osteoblasts suppresses osteoclastogenesis via inhibiting IL-6 expression. Bone Rep. 2019;11:100228. doi:10.1016/j.bonr.2019.100228

18. Hu X, Liu H, Wang Z, Hu Z, Li L. miR-200a attenuated doxorubicin-induced cardiotoxicity through upregulation of Nrf2 in mice. Oxid Med Cell Longev. 2019;2019:1512326. doi:10.1155/2019/ 1512326 
19. Wang L, Zhuang L. Astaxanthin ameliorates the lipopolysaccharides-induced subfertility in mouse via Nrf2/HO-1 antioxidant pathway. Dose Response. 2019;17(3):15593258 19878537. doi: $10.1177 / 1559325819878537$

20. Shi S, Lei S, Tang C, Wang K, Xia Z. Melatonin attenuates acute kidney ischemic reperfusion injury in diabetic rats by activation of the SIRT1/Nrf2/HO-1 signaling pathway. Biosci Rep. 2018;39(1).

21. Weng J, Zhou J, Liang L, Li L. UHPLC/QTOF-MS-based metabolomics reveal the effect of Melastoma dodecandrum extract in type 2 diabetic rats. Pharm Biol. 2019;57(1):807-815. doi:10.1080/ 13880209.2019 .1693605

22. Hu B, Tong F, Xu L, et al. Role of calcium sensing receptor in streptozotocin-induced diabetic rats exposed to renal ischemia reperfusion injury. Kidney Blood Press Res. 2018;43(1):276-286. doi: $10.1159 / 000487685$

23. Yan Y, Bai J, Zhou X, et al. P2X7 receptor inhibition protects against ischemic acute kidney injury in mice. Am J Physiol Cell Physiol. 2015;308(6):C463-72. doi:10.1152/ajpcell.00245.2014

24. Fouad AA, Al-Mulhim AS, Jresat I, Morsy MA. Protective effects of captopril in diabetic rats exposed to ischemia/reperfusion renal injury. $J$ Pharm Pharmacol. 2013;65(2):243-252. doi:10.1111/j.20427158.2012.01585.x

25. Tawfik MK. Renoprotective activity of telmisartan versus pioglitazone on ischemia/reperfusion induced renal damage in diabetic rats. Eur Rev Med Pharmacol Sci. 2012;16(5):600-609.

26. Zhang Y, Yang S, Cui X, et al. Hyperinsulinemia can cause kidney disease in the IGT stage of OLETF rats via the INS/IRS-1/PI3-K/Akt signaling pathway. J Diabetes Res. 2019;2019:4709715. doi:10.1155/ 2019/4709715

27. Yan Z, Zang B, Gong X, Ren J, Wang R. MiR-214-3p exacerbates kidney damages and inflammation induced by hyperlipidemic pancreatitis complicated with acute renal injury. Life Sci. 2019;241:117118.
28. Tong Y, Liu S, Gong R, Zhong L, Duan X, Zhu Y. Ethyl vanillin protects against kidney injury in diabetic nephropathy by inhibiting oxidative stress and apoptosis. Oxid Med Cell Longev. 2019;2019:2129350. doi:10.1155/2019/2129350

29. Liu SS, Chen YY, Wang SX, Yu Q. Protective effect of dabrafenib on renal ischemia-reperfusion injury in vivo and in vitro. Biochem Biophys Res Commun. 2019.

30. Dong Q, Jie Y, Ma J, Li C, Xin T, Yang D. Renal tubular cell death and inflammation response are regulated by the MAPK-ERK-CREB signaling pathway under hypoxia-reoxygenation injury. $J$ Recept Signal Transduct Res. 2019;39(5-6):383-91.

31. Chang JF, Yeh J-C, Ho C-T, et al. Targeting ROS and cPLA2/COX2 expressions ameliorated renal damage in obese mice with endotoxemia. Int J Mol Sci. 2019;20(18):4393. doi:10.3390/ijms20184393

32. Chen H, Wan D, Wang L, et al. Apelin protects against acute renal injury by inhibiting TGF- $\beta 1$. Biochim Biophys Acta. 2015;1852 (7):1278-1287. doi:10.1016/j.bbadis.2015.02.013

33. Sagiroglu T, Torun N, Yagci M, Yalta T, Sagiroglu G, Oguz S. Effects of apelin and leptin on renal functions following renal ischemia/ reperfusion: an experimental study. Exp Ther Med. 2012;3 (5):908-914. doi:10.3892/etm.2012.499

34. Amini N, Sarkaki A, Dianat M, Mard SA, Ahangarpour A, Badavi M. Protective effects of naringin and trimetazidine on remote effect of acute renal injury on oxidative stress and myocardial injury through Nrf-2 regulation. Pharmacol Rep. 2019;71(6):1059-1066. doi:10.1016/j.pharep.2019.06.007

35. Shen X, Hu B, Xu G, et al. Activation of Nrf2/HO-1 pathway by glycogen synthase kinase- $3 \beta$ inhibition attenuates renal ischemia/ reperfusion injury in diabetic rats. Kidney Blood Press Res. 2017;42 (2):369-378. doi:10.1159/000477947

Diabetes, Metabolic Syndrome and Obesity: Targets and Therapy

Dovepress

\section{Publish your work in this journal}

Diabetes, Metabolic Syndrome and Obesity: Targets and Therapy is an international, peer-reviewed open-access journal committed to the rapid publication of the latest laboratory and clinical findings in the fields of diabetes, metabolic syndrome and obesity research. Original research, review, case reports, hypothesis formation, expert opinion and commentaries are all considered for publication. The manuscript management system is completely online and includes a very quick and fair peer-review system, which is all easy to use. Visit http://www.dovepress.com/testimonials.php to read real quotes from published authors. 